International Conference on Renewable Energies and Power Quality (ICREPQ'11)

Las Palmas de Gran Canaria (Spain), 13th to 15th April, 2011

\title{
Design and test of a 300Wh composites flywheel energy storage prototype with active magnetic bearings
}

\author{
Xingjian DAI, Kai ZHANG and Xiao-zhang ZHANG \\ Department of Engineering Physics \\ Tsinghua University \\ Beijing, 100084 (P. R. China) \\ Phone/Fax number:+0086 10 62784518/+008610 62782658, e-mail: daixj@mail.tsinghua.edu.cn
}

\begin{abstract}
A flywheel energy storage prototype was designed and built to get high energy density and low bearing loss. The aluminium alloy hub formed by thin plate and shell connected the bearing shaft and the rim which was composed by glass fibre and carbon fibre reinforced composite. Finite element analysis on stress field of the composite flywheel indicated that the flywheel could run at the rated speed of $700 \mathrm{r} / \mathrm{s}$ (rotation per second) safely. The flywheel was supported by 5-degrees of freedom active magnetic bearings (AMBs). The flexible modes of the rimhub-shaft system were analyzed by using finite element (FE) based software. The complex non-synchronous vibration was observed, analyzed and suppressed during testing of the flywheel system.

The control method of adding phase compensator to the velocity channel in the cross feedback controller was presented to make the supporting AMBs work better while the flywheel passed through its first flexible mode. The field balancing at high speeds enabled the flywheel to reach the speed of $475 \mathrm{r} / \mathrm{s}$ with small amplitude of synchronous response.
\end{abstract}

\section{Key words}

Flywheel energy storage; Composites; Active magnetic bearings

\section{Introduction}

Application of high speed flywheels as mechanical batteries to store energy becomes increasingly attractive in recent years [1]. In opposition to chemical batteries, the life of AMB flywheels has no degradation during the entire design life. A spinning flywheel has capability to accumulate a large amount of kinetic energy. Therefore, having an integrated motor/generator, the well controlled flywheel can be used not only for the attitude control but also for energy storage in spacecraft $[2,3]$.
One of the main sources of energy loss in flywheels is attributed to bearings. With the introduction of frictionless active magnetic bearings, the efficiency of flywheels for energy storage has been increased to an economically useful level. Electrical active magnetic bearings (AMBs) satisfy the frictionless condition but require advanced control systems [4, 5]. Although the super-conducting bearings have been developed to replace conventional bearings [6, 7], they require cryogenic conditions, and $\mathrm{AMBs}$ still remain the best bearing alternative for flywheels in spacecraft application.

To demonstrate the possible space application of the advanced composite flywheel with AMBs, the experimental high speed (in rated rotational frequency of $700 \mathrm{~Hz}$ ) FES-AMB system was designed and built in Tsinghua University. The stress and stain field of the flywheel was solved by FEM, which indicated that the structural failure would not happen while the rotational speed being up to $700 \mathrm{r} / \mathrm{s}$. It was found difficult to use the conventional cross feed back control to make the flywheel stable when it passes through the resonant vibration district because of the flexible mode due to the flexible hub connection between the shaft and the rim. Compared with the past literature, the new challenge is the coupling between the strong gyroscopic and the flexible mode resonance at the high speed around $400 \mathrm{r} / \mathrm{s}$.

The complex nonsynchronic vibration behaviour was observed and analyzed during experimental testing of the flywheel system with flexible components. Several control methods were successfully applied to suppress the unexpected modal vibrations. The AMB controller was revised and substantially improved to guarantee stable operation when the flywheel passed two rigid modes and the first flexible mode. 


\section{Flywheel Structural Analysis}

\section{A. Flywheel AMBs System}

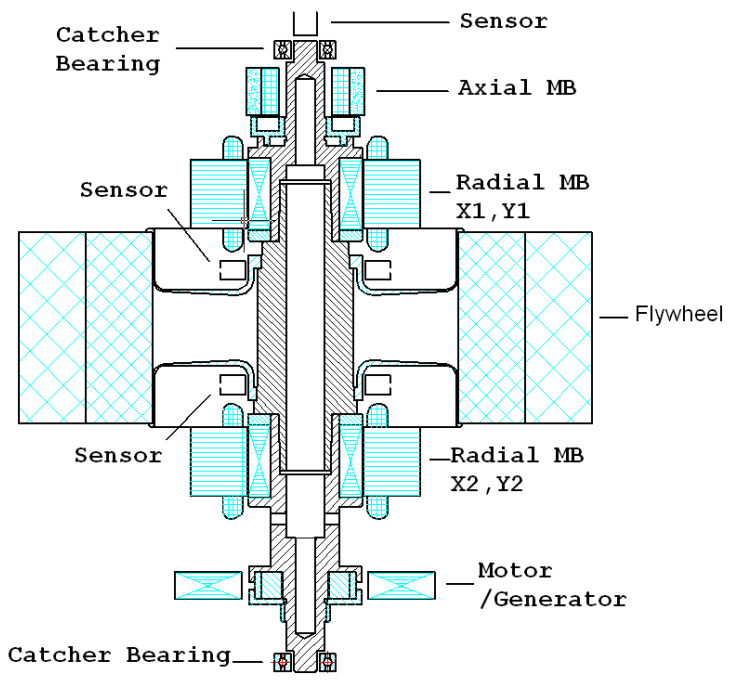

Fig. 1 Flywheel AMBs system

The flywheel levitated by magnetic forces in five degrees of freedom is shown in Fig. 1. The composite rim is connected to the shaft through the aluminum hub. The radial and axial magnetic forces act on the shaft to levitate the flywheel. The flywheel AMBs system has two rotordynamic characteristics. One is the strong gyroscopic effect due to high speed and the large polar moment of inertia, and the other one is the flexible modes in frequency lower than the rated rotational frequency of 700 $\mathrm{Hz}$, which is due to the fact that the flywheel has hub with low rigidity.

Table 1 Parameters of the flywheel

\begin{tabular}{l|l}
\hline Mass & $11.9 \mathrm{~kg}$ \\
\hline Diameter & $300 \mathrm{~mm}$ \\
Length & $100 \mathrm{~mm}$ \\
Polar momean of inertia & $0.13 \mathrm{kgm}^{2}$ \\
Diameter moment of inertia & $0.087 \mathrm{kgm}^{2}$ \\
Rate speed & $700 \mathrm{r} / \mathrm{s}$ \\
\hline Sotred enery & $340 \mathrm{Wh}$ \\
\hline
\end{tabular}

\section{B. Rim-hub Stress Analysis}

One of main concern of flywheel technology is how to improve the flywheel rotating speed due to the energy stored being proportional to the square of the speed. The specific energy is proportional to the specific strength of materials. Therefore, the fiber-reinforced polymer composites with high specific strength are usually used as the rim materials [8]. The composite rim is attached to a metal shaft through a metallic web or spoke hub which compensates the deformation difference between the rim and the shaft under the high speed running condition. The design procedure was discussed in reference [9].

In the detail design, the finite element analysis is employed to know the stress and strain field of the flywheel composition. The composite rim is either press-fit or shrink-fit to the metal hub due to the deformation of the rim is much greater than the hub under centrifugal load. In our test flywheel case, the shrink-fit size is $0.30 \mathrm{~mm}$. The composites rim is composed by two rings by winding fabrication method. The inner ring is glass fiber reinforced polymer (GFRP) and the outer ring is carbon fiber reinforced polymer (CFRP).

Talbe 2 Mechanics parameters of flywheel materials[9]

\begin{tabular}{lcccccc}
\hline & $E_{x}$ & $E_{z}$ & $\rho$ & $\sigma_{L S}$ & $\sigma_{T S}$ & $\sigma_{b}$ \\
& $\mathrm{GPa}$ & $\mathrm{MPa}$ & $\mathrm{kg} \cdot \mathrm{m}^{-3}$ & $\mathrm{MPa}$ & $\mathrm{MPa}$ & $\mathrm{MPa}$ \\
\hline GFRP & 15 & 65 & 2100 & 1600 & 30 & - \\
CFRP & 7 & 130 & 1550 & 1800 & 20 & - \\
7050 & 72.5 & 72.5 & 2800 & - & - & 570 \\
\hline
\end{tabular}

The plane axial-symmetrical model was used to calculate the stress of flywheel under rotation centrifugal load in FEM analysis. In the elastic calculation, the first load step is shrink-fit size being $0.3 \mathrm{~mm}$, the second load step is centrifugal load under the rotational speed of $700 \mathrm{r} / \mathrm{s}$. The highest Von Mises stress of the aluminum alloy hubs is $330 \mathrm{MPa}$, being lower than the stress limit of aluminum alloy 7050. The maximum stress value of the composites rim was shown in Table 3 . The rim materials are safe while the maximum stress failure criterion could be applied.

Table 3 The maximum stress in rim

\begin{tabular}{lccccc}
\hline Composite & $\sigma_{r} / \mathrm{MPa}$ & $\sigma_{\theta} / \mathrm{MPa}$ & $\sigma_{z} / \mathrm{MPa}$ & $\sigma_{L S} / \mathrm{MPa}$ & $\sigma_{T S} / \mathrm{MPa}$ \\
\hline GFRP & 19.4 & 470 & 6.9 & 1700 & 30 \\
CFRP & 13.1 & 617 & 4.1 & 2300 & 20 \\
\hline
\end{tabular}

Fig. 2 displayed the radial displacement of hub. The hub will expand $0.31 \mathrm{~mm}$ in radial direction. Fig. 3 indicated that there are enough press force between the hub and the rim, which make sure the two parts keeping in fixed contact.

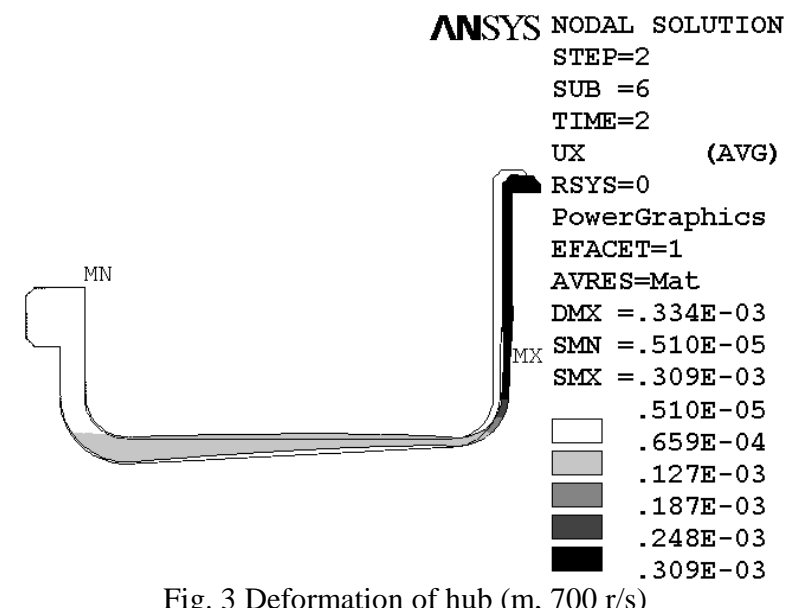

Fig. 3 Deformation of hub (m, $700 \mathrm{r} / \mathrm{s})$ 


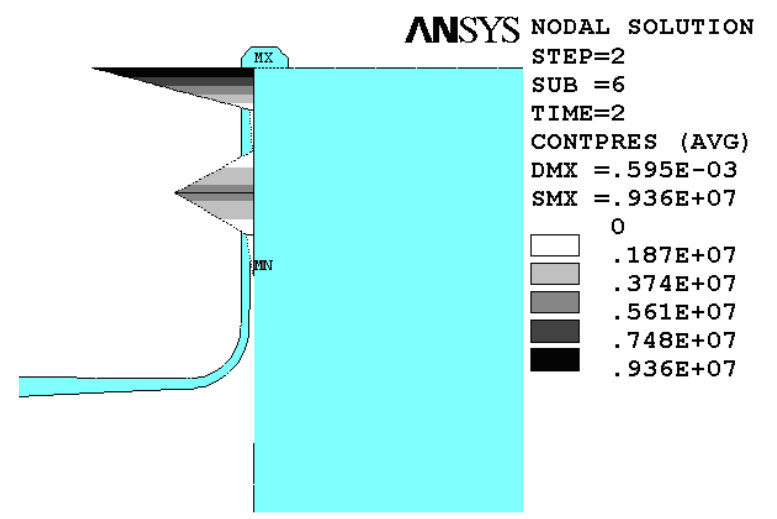

Fig. 3 Rim-hub contact pressure $(\mathrm{Pa}, 700 \mathrm{r} / \mathrm{s})$

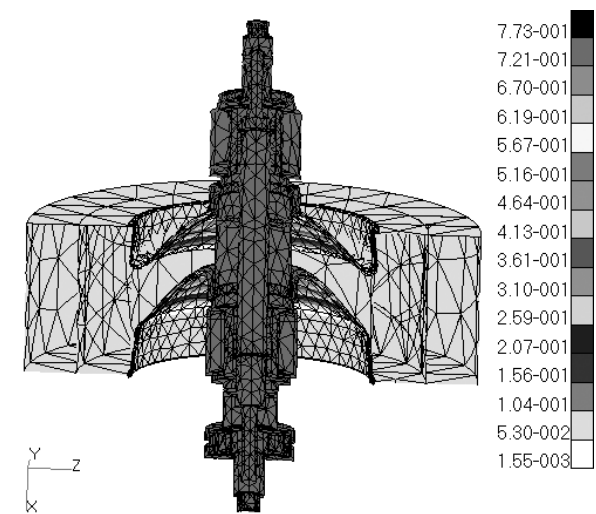

Hub axial mode

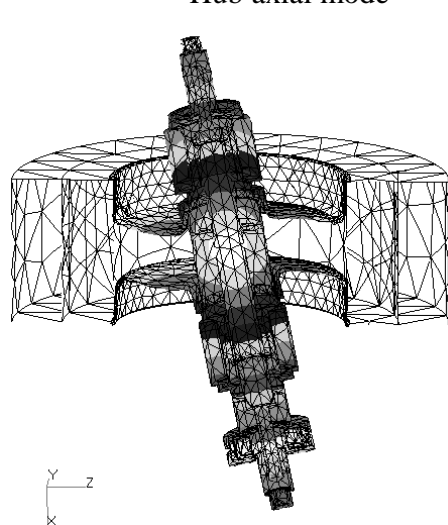

$1.93+000$
$1.80+000$
$1.68+000$
$1.55+000$
$1.42+000$
$1.29+000$
$1.16+000$
$1.03+000$
$9.03-001$
$7.74-001$
$6.45-001$
$5.16-001$
$3.87-001$
$2.59-001$
$1.30-001$
$8.12-004$

Hub deflection mode

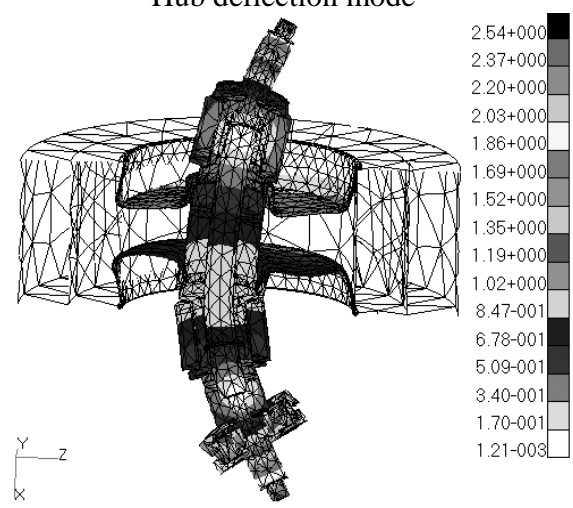

Shaft curved mode

Fig.4 Flexible hub-rim-shaft modes

\section{Modal Vibration Analysis}

\section{A. Calculation}

In the simplified modal vibration analysis of flywheelbearing system, the flywheel could be considered as a rigid disk locating at the middle of a rigid shaft supported on active magnetic bearings. The performance of the precession in lower frequency and the nutation in high frequency split a lot with the growing rotating frequency due to gyroscopic effect of the flywheel in thin disk form. The splitting modes make the system be parameterdependent and pose a problem for the AMB controller. The splitting modes will behave as the sub-harmonic and supper-harmonic vibration when the modal damping become decaying as the flywheel speed growing up.

The hub with good ability of deformation has the function of connection between the composite rim and the metal core shaft. However, the stiffness of the thin shell is not high enough to make the flexible modal frequency of the rim-hub-shaft system being bigger than the rated rotational frequency $(700 \mathrm{~Hz})$.

For such a system composed by rim, shell hub, metal core shaft and bearings, the finite element methods can be quite useful to obtain its vibration mode parameters. In this research ANSYS package has been employed to reveal the flexible modes of the flywheel in its nonrotating state. The analysis indicated that there were three flexible modes with frequency under $1000 \mathrm{~Hz}$. The first is a "hub axial" mode, when the hub deforms mainly in axial direction (see Fig. 4), with the frequency of 225.8 Hz. The second is the "hub deflection" mode, as shown in Fig. 4, at the frequency $332.4 \mathrm{~Hz}$. The last modal frequency is $937.5 \mathrm{~Hz}$, where the modal shape is expressed as the bending of the core shaft. (see Fig. 4).

\section{B. Identification of Modal Frequency}

Both the impact test and the force excitation via AMB were used to extract natural frequencies. In the impact test, the impact force was acted on the flywheel-shaft in freely suspended state along both the radial and axial direction separately by the hamper. The sine sweep force excitation test is convenient and useful to obtain the vibration performance of the AMB supported rotors because the excitation and the response can be easily measured.

Table 2 Comparison of the modal frequency / $\mathrm{Hz}$

\begin{tabular}{l|lll}
\hline Mode & $\begin{array}{l}\text { Hub } \\
\text { axial }\end{array}$ & $\begin{array}{l}\text { Hub } \\
\text { deflection }\end{array}$ & $\begin{array}{l}\text { Core shaft } \\
\text { bending }\end{array}$ \\
\hline $\begin{array}{l}\text { Calculated } \\
\text { results }\end{array}$ & 225.8 & 328.4 & 917.3 \\
$\begin{array}{l}\text { Impact test } \\
\text { Electric- } \\
\text { magnetic } \\
\text { excitation }\end{array}$ & 210 & 320.0 & 900 \\
\hline
\end{tabular}

To arrive at the speed of 700rps, the flywheel has to pass through the three resonance speeds, two rigid (i.e., cylindrical and conical) and the flexible hub deflection. The hub axial mode was not considered because there is no obvious excitation along the axial direction. The first flexible modal frequency $(328 \mathrm{~Hz})$ of the hub increases greatly because of strong flywheel gyroscopic effects. 
The possible three kinds of nonsynchronous modal vibrations may occur during the start-up process.

\section{Nonsynchronous Vibrations}

\section{A. Levitation at Zero Speed}

The very basic requirement for $\mathrm{AMB}$ rotor systems is to achieve stable levitation, where the shaft remains in concentric position at the bearings.

The high order modal vibration was found in the static levitation, which could be attenuated by adding damping in the controller. The frequency of $350 \mathrm{~Hz}$ is attributed to hub deflection mode, which can be lowered by the loop shaping technique which means to add additional filter into the controller to change the phase and gain characters at a local frequency range. The third frequency of $950 \mathrm{~Hz}$ in peak response value belonged to the first flexible shaft mode, which can be damped by adding a notch filter in the controller. The improved PID controller at zero speed made the maximum vibration amplitude being lower than $1 \mu \mathrm{m}$.

\section{B. Subharmonic Vibration}

After the stable levitation at zero speed is achieved, the flywheel dynamics should be tested during the speeding up. Complex vibration phenomenon was observed during this process. Figure 5 presents large subharmonic vibration measured at the running speed of $82 \mathrm{r} / \mathrm{s}$. The center of the flywheel follows an unstable trajectory. Spectrum analysis indicates that this is the natural backward precession in low frequency of $8 \mathrm{~Hz}$. The subharmonic vibration amplitude may inflate and make the shaft to contact the backup bearings.
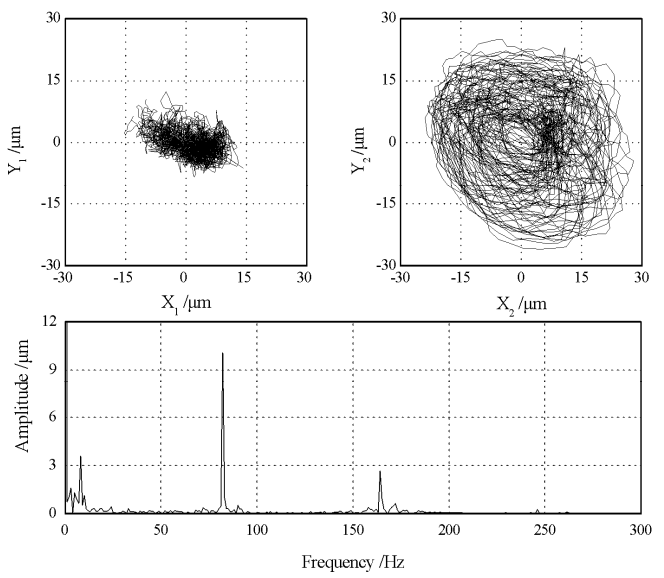

Fig. 5 The precession in low frequency
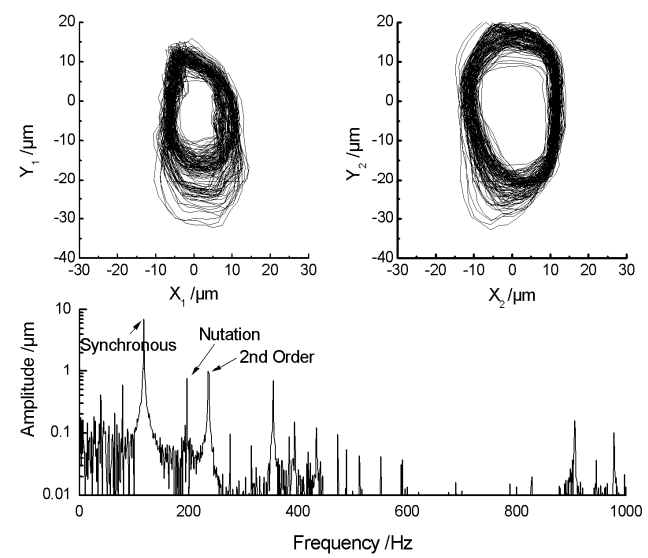

Fig. 6 The nutation in high frequency

\section{Superharmonic Vibration}

The running of flywheel with strong gyroscopic effect caused instability in the flywheel-hub-shaft bearings system. The PID controller could not confirm the stable running of the flywheel in speeding up. The cross feedback control techniques were presented to lead better system performance [10].

Under the positive displacement cross feedback control, the flywheel has been run to $119 \mathrm{r} / \mathrm{s}$ without subharmonic vibration. However, the superharmonic vibration occurred at this time. The spectrum components seem to be very complex. Fig.6 indicates that the superharmonic motion is mainly composed by the $2^{\text {nd }}$ order vibration and the nutation. The nutation could be suppressed by the minus cross feedback in the displacement channel and the minus cross feedback in the velocity channel. On the other hand, the positive displacement cross feedback would suppress the precession but excite the nutation. Therefore, the displacement cross feedback factor must be select carefully. Considering the precession frequency being very low and the nutation frequency being very high, it will get better results to add a low-pass filer into the positive displacement cross feedback channel and add a high-pass filter into the minus displacement cross feedback channel [11].

The elaborate combined application of the low-pass or high-pass filter and the positive or minus displacement cross feedback produced good result which exhibited as the smooth operation of the flywheel up to 280rps.

\section{Flexible Modal Vibration}

Both the subharmonic precession and the super-harmonic nutation discussed above were rigid modes of the flywheel-bearing system. The flywheel keeps rigid body state. The other kind of super-harmonic vibration can be the flexible shell mode vibration. Fig. 7 shows the observed 3 times harmonic vibration whose frequency is attributed to the flexible hub radial mode. The excitation source was from the bearings' electromagnetic forces with components in high frequency. In the application of "loop shaping" method as mentioned above, the adjusting parameters were selected to get better mode damping of 
the flexible rub radial mode. The flywheel was running up to $170 \mathrm{r} / \mathrm{s}$ without the flexible modal vibration.
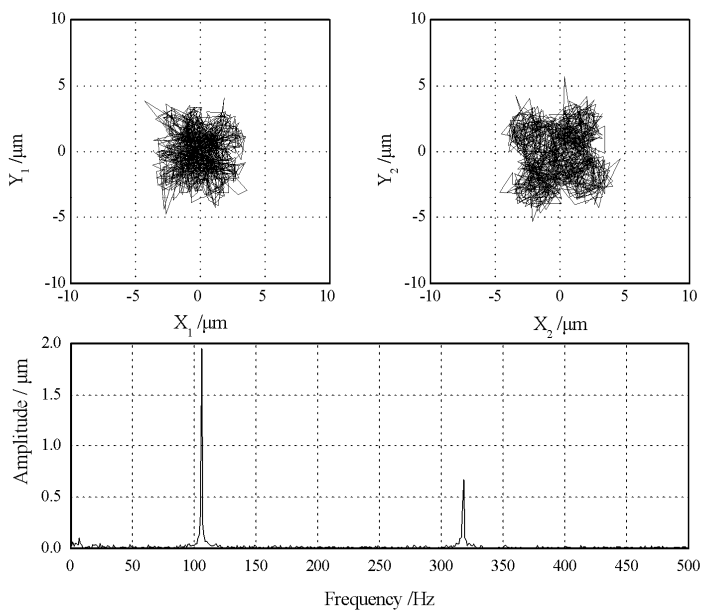

Fig. 7 The flexible modal vibration at low speed
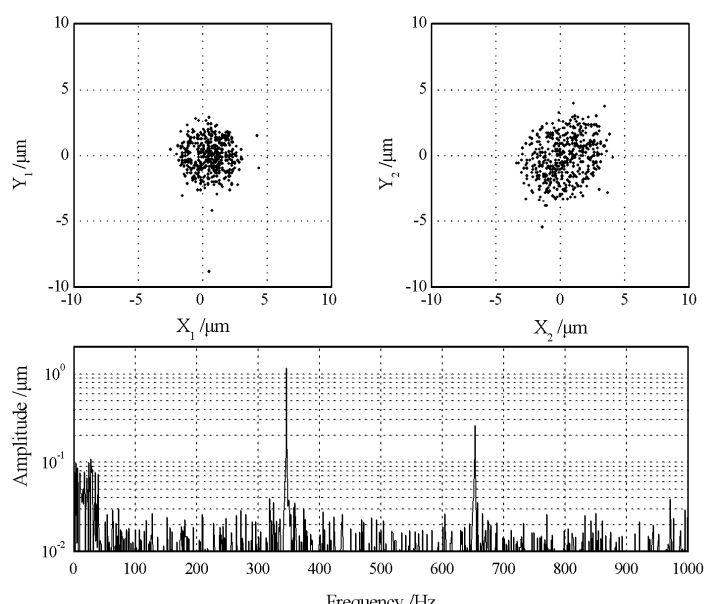

Fig. 8 The flexible modal vibration at high speed

\section{Passing through the Flexible Critical Speed}

The dynamics analysis indicated that the flywheel-ABMs system should pass through the flexible critical speed as high as $400 \mathrm{r} / \mathrm{s}$. The nutation was observed again as the flywheel run to $346 \mathrm{r} / \mathrm{s}$ (see Fig. 8). To suppress the nutation near the flexible modal resonance, the "phase compensator" was added to the velocity cross feedback channel of the controller. The phase compensator alters the gain-frequency and phase-frequency characters of the velocity cross feedback channel and provides additional phase lead for the controller. Then, the flywheel ran to 360 r/s smoothly.

Although AMBs have the ability to compensate the unbalance of the rotor, the unbalance compensation scheme was not used because the concern was focused on the suppression of the nonsychronous vibration. The flywheel-AMB system was balanced in the field. The twoplane-effect-coefficient balancing method was employed to achieve smooth speeding up with residual unbalance response (seeing Fig. 9). Several balancing experiments were conducted at speeds 130, 166, 257 and 300r/s.

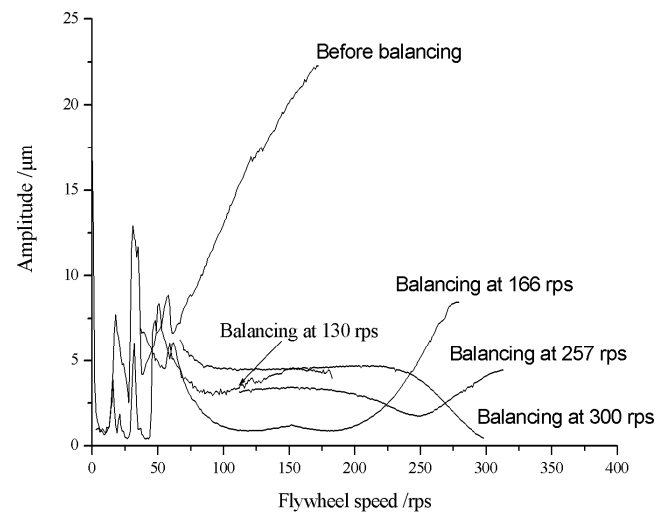

Fig. 9 Balancing at different speeds

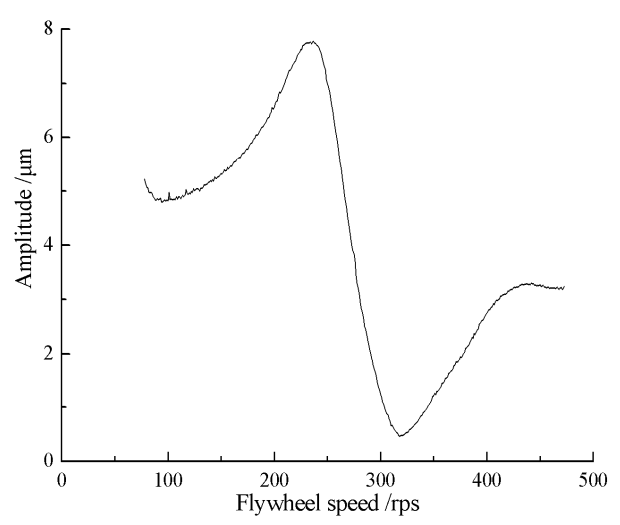

Fig. 10 The unbalance response in high speed running

After numerous trials with the controller, and careful balancing, the flywheel was able to pass the critical speed of $420 \mathrm{r} / \mathrm{s}$ and run up to the speed of $475 \mathrm{r} / \mathrm{s}$, as shown in Fig. 10. The AMB flywheel was not run at higher speeds because that the temperature became very high due to both the wind loss in the chamber without high vacuum and the heating from the eddy current loss of the magnetic bearings. The improvement of the vacuum condition and the lower power control of AMBs should be studied further to enable the flywheel run to the rated speed of $700 \mathrm{r} / \mathrm{s}$ in the future.

\section{Conclusion}

The structural stress analysis indicated that the material failure would not happen when the flywheel was speeded up to $700 \mathrm{r} / \mathrm{s}$. The modal analysis of the AMB flywheel system indicated that both the rigid modes and the flexible modes were presented in the system. The flexible mode manifested as the hub deflection shape because of the application of the thin aluminium shell with low stiffness. The prediction was similar to the test results. Several control methods such as the loop shaping, the notch filter, and the cross feedback were combined to control the nonsynchronous vibrations of the flywheelAMBs system. The new control method of adding phase compensator to the velocity cross feedback channel was presented and it made AMBs performing well while the flywheel passes the high critical speed at the first flexible 
mode. The in-field balancing at high speeds made the flywheel run to the speed of $475 \mathrm{r} / \mathrm{s}$ with small synchronous response.

\section{References}

[1] P. J. Hall, E. J. Bain, "Energy-storage technologies and electricity generation", Energy Policy, 2008, Vol. 36, pp. 4352-4355.

[2] Renuganth Varatharajoo, "A combined energy and attitude control system for small satellites", Acta Astronautica 2004, Vol. 54, pp. 701-712.

[3] B. H. Kenny, "Control of a high-speed flywheel system for energy storage in space applications", IEEE transactions on industry applications, 2005,Vol. 41(4), pp. 1029-1038.

[4] C.R. Knospe, "Active magnetic bearings for machining applications", Control Engineering Practice, 2007, Vol. 15, pp. 307-313.

[5] J. Shen, B.C. Fabien, "Optimal control of a flywheel energy storage system with a radial flux hybrid magnetic bearing", Journal of Franklin Institute, 2002, Vol. 339, pp. $189-210$

[6] Mulcahe, T. M., Hull, J. R., Uherke, K. L. etc. "Flywheel energy storage advanced using HTS bearings", IEEE Transactions on Applied Superconductivity, 1999, Vol. 9(2), pp. 297-300.

[7] O. Tsukamoto, A. Utsunomiya, "HTS flywheel energy storage system with rotor shaft stabilized by feed-back control of armature currents of motor-generator", Physica C: Superconductivity and its Applications, 2007, Vol. 463465, n SUPPL., pp. 1267-1270.

[8] J. Tzeng, R. Emerson and P. Moy, "Composite flywheel for energy storage", Composites Science and Technology, 2006, Vol. 66, pp. 2520-2527.

[9] X. Dai, Y. Li and H. Yu, "Design of high specific energy density flywheel"(in Chinese), Journal of Tsinghua University, 2008, Vol. 48, pp. 379-382.

[10] M. Ahrens, L. Kuceara, and R. Larsonner, "Performance of a magnetically suspended flywheel energy storage device", IEEE Transactions on Control system Technology, 1996, Vol.4, pp. 494-502.

[11] J.G. Bai, Investigation of active magnetic bearings for flywheel energy storage system. Doctor dissertation (in Chinese), Tsinghua University, Beijing, P.R. China. 2007. 